\title{
Abordaje de lesiones cavitadas pulmonares. Lesión quística pulmonar resuelta por toracoscopia. Reporte de caso
}

\author{
Víctor Hugo Zotes-Valdivia, $\bowtie$ Marco Antonio Iñiguez-García, Cesar Luna-Rivero, \\ José Luis Téllez-Becerra
}

Instituto Nacional de Enfermedades Respiratorias Ismael Cosío Villegas, Ciudad de México.

Trabajo recibido: 01-X-2014; aceptado: 27-I-2015

\begin{abstract}
RESUMEN. El concepto de lesión pulmonar cavitada y quiste es diferente. Un minucioso análisis clínico y radiológico de ambos procesos puede limitar de gran manera las posibilidades diagnósticas. Presentamos el caso de una mujer de 21 años de edad ingresada por una lesión quística pulmonar, la cual, tras el reporte histopatológico, se concluyó como un absceso pulmonar crónico, el mismo que fue resuelto por resección toracoscópica. Los abscesos pulmonares son lesiones intraparenquimatosas cavitadas formados por un proceso infeccioso secundario a la introducción en el árbol bronquial, de una carga microbiológica importante. De acuerdo al tiempo de evolución son clasificados en agudos o crónicos. El tratamiento está basado en la identificación oportuna del germen e inicio de terapia antimicrobiana. El drenaje percutáneo junto a una apropiada terapia antimicrobiana, ha reducido la necesidad de intervenciones quirúrgicas por esta causa, limitándose sólo a indicaciones precisas. El desarrollo tecnológico ha permitido la introducción de las técnicas de mínima invasión a la cirugía torácica. Las ventajas de esta técnica están relacionadas principalmente a la disminución del dolor posoperatorio y la reducción de los días de hospitalización.
\end{abstract}

Palabras clave: Lesión cavitada pulmonar, absceso pulmonar crónico, tratamiento quirúrgico, toracoscopia.

ABSTRACT. The concept of cavitary lung lesion and cyst is different. The clinical and radiological analysis of both processes can greatly limit the diagnostic possibilities. Lung abscess are cavitated intraparenchymal lesions formed by a secondary infection process for the introduction into the bronchial tree of important microbiological burden. According to the duration of symptoms are classified into acute or chronic. Treatment is based on the timely identification of microbiological agent and beginning of antimicrobial therapy. Percutaneous drainage with appropriate antimicrobial therapy has reduced the need for surgical intervention; in fact, it is only indicated to specific situations. Technological development has enabled the introduction of minimally invasive techniques in thoracic surgery. The advantages of this technique are mainly related to the reduction of postoperative pain and reducing hospital days, compared to lower cost offered by traditional surgery, the latter seems to be equal in both over time. While the literature is extensive referring to lung abscess, is not the case when it comes to lung abscess in chronic stage. We report a 21-years old woman with histopathological diagnosis of chronic lung abscess, treated by thoracoscopic resection.

Key words: Cavitary lung lesion, chronic lung abscess, surgical treatment, thoracoscopy.

\section{INTRODUCCIÓN}

De acuerdo a la literatura radiológica, una cavitación es un espacio lleno de gas, visto como un área de lucencia o de baja atenuación, dentro de una consolidación pulmonar, una masa, o un nódulo. En el caso de consolidación cavitada, la consolidación original puede resolver y dejar sólo una pared delgada. Una cavitación normalmente es producida por la expulsión o el drenaje de la parte necrótica de la lesión a través del árbol bronquial. A veces contiene un nivel líquido. Cavitación no es sinónimo de absceso. ${ }^{1}$

Por otro lado, un quiste pulmonar se define como un espacio parenquimatoso pulmonar anormal, relleno de aire o líquido, con una pared de grosor variable, delgada o gruesa y recubierta por epitelio. Desde el punto de vista radiológico, el quiste puede definirse como un espacio que contiene aire, de bordes bien definidos y pared fina; según Naidich et al., ésta debe ser menor de $3 \mathrm{~mm}$ y de tamaño variable. ${ }^{2}$

Se ha tratado de hacer la distinción entre quiste y cavidad tomando en cuenta el grosor de su pared, definiendo un quiste como un espacio aéreo rodeado de una pared de $4 \mathrm{~mm}$ o menos de grosor; y una cavidad, con una pared de $5 \mathrm{~mm}$ o más gruesa. ${ }^{3}$ Desafortunadamente, existe una gran variedad de lesiones que se sobreponen a estas dos categorías, lo cual no hace 
posible categorizar estos dos tipos de lesión tan sólo por el grosor de pared.

Los abscesos pulmonares son lesiones intraparenquimatosas cavitadas formados por un proceso infeccioso secundario a la introducción en el árbol bronquial de una carga microbiológica importante (broncoaspiración), o al fracaso de los mecanismos de depuración microbiológica en los pulmones (obstrucción bronquial). La clasificación de los abscesos pulmonares está relacionada con el tiempo de evolución: abscesos agudos tienen menos de cuatro a seis semanas de evolución. Abscesos crónicos con más de seis semanas de inicio. ${ }^{4}$ Además, según la ausencia o presencia de factores predisponentes, éstos pueden ser primarios o secundarios, respectivamente.

La cirugía torácica no ha estado ajena a la revolución tecnológica de la cirugía, tan es así que a partir de 1990 los artículos acerca de nuevos procedimientos toracoscópicos han aumentado en todas las publicaciones de la especialidad. Es necesario recordar que esta modalidad terapéutica fue descrita por Jacobeus en 1910, quien mediante un cistoscopio practicó por primera vez una inspección de la cavidad pleural. Aunque en un comienzo su intención era solamente diagnóstica, describió en 1921 una técnica para dividir adherencias pleurales mediante un galvanocauterio, lo que hacía posible la formación de neumotórax terapéutico y obliteración de cavernas tuberculosas. Luego, en 1922 publicó una técnica para liberar adherencias en forma extrapleural y destacó que es posible diferenciar entre tumor y tuberculosis mediante el uso del toracoscopio, y corroborar esto mediante biopsias y estudio histopatológico. ${ }^{5}$

A su vez, Lob y Weiss, en 1952, demostraron la utilidad de la toracoscopia para establecer la causa en derrames pleurales recidivantes, cuyos estudios citológicos y de biopsia por aguja eran negativos. Touraine, en la década de los sesenta describió los hallazgos normales en la pleura parietal y visceral cuando se inspecciona mediante el toracoscopio.

Maasen efectuó 40 biopsias pulmonares entre 1970 y 1972 mediante un mediastinoscopio, con el cual podía inspeccionar la pleura y tomar una parte del pulmón, los que eran exteriorizados juntos al momento de retirar el instrumento, pudiendo así tomar la muestra de pulmón en forma extratorácica. ${ }^{6}$

Como puede apreciarse, los avances en los últimos 70 años han sido constantes; pero sin lugar a dudas, ha sido el desarrollo tecnológico de la óptica y el diseño de nuevas microcámaras, los que han hecho posible anexar un equipo de video a los toracoscopios tradicionales. Lo anterior, junto al diseño y creación de instrumental adecuado, han permitido aumentar el espectro de intervenciones que se pueden efectuar dentro del tórax en forma endoscópica.

Presentamos el caso de una mujer de 21 años, quien ingresó al Servicio de Neumología para el estudio de una lesión pulmonar, radiológicamente de apariencia quística, pero con diagnóstico histopatológico de absceso pulmonar crónico, localizado en el lóbulo superior derecho, y que se benefició de una resección toracoscópica.

\section{Observación clínica}

Mujer de 21 años que ingresó por cuadro de infecciones recurrentes de vía aérea superior y disnea de seis meses de evolución. En retrospectiva, refirió presencia de tos persistente con expectoración verde amarillenta, además, de alzas térmicas no cuantificadas al inicio de su padecimiento, por lo que recibió múltiples esquemas antimicrobianos no especificados durante un período aproximado de dos meses, manifestando leve mejoría. La exploración física fue anodina.

Se decidió su ingreso por hallazgo radiológico de lesión radiolúcida redondeada de pared radiopaca delgada y bien definida en hemitórax derecho (figura 1A). La tomografía de tórax de alta resolución reveló una lesión ocupante de apariencia quística en hemitórax derecho, redondeada, hipodensa, de paredes bien definidas, midió aproximadamente $6 \times 5 \mathrm{~cm}$ de diámetro y $3 \mathrm{~mm}$ de grosor de pared, que aparentó comunicación con la vía aérea (figura 1B). La espirometría fue normal (FEV $184 \%$, FVC 88\% y FEV $/$ /FVC 82\%). La biometría hemática, química sanguínea, electrolitos, perfil hepático y pruebas de coagulación sin alteraciones. Serología para HIV negativa.

Con alta sospecha diagnóstica de lesión quística pulmonar de origen a determinar, o probable enfermedad pulmonar bullosa, se decidió resección quirúrgica por mínima invasión.

\section{Técnica quirúrgica}

Se realizó abordaje toracoscópico a tres puertos: uno para la cámara en el sexto espacio intercostal sobre la línea axilar posterior, los otros dos puertos de trabajo en el quinto espacio sobre la línea axilar media y subescapular. Se identificó la lesión intraparenquimatosa dependiente de lóbulo superior con adherencias laxas a pared y a lóbulo medio, las cuales se liberaron con electrocauterio. Se procedió a disección completa de la lesión y resección con engrapadora (figura 2), quedando fugas de tipo alveolar en lóbulo medio por la contigüidad a la lesión. El sangrado aproximado fue menor de $50 \mathrm{~mL}$. Se dejó un drenaje blando de silicón de 24 Fr. 

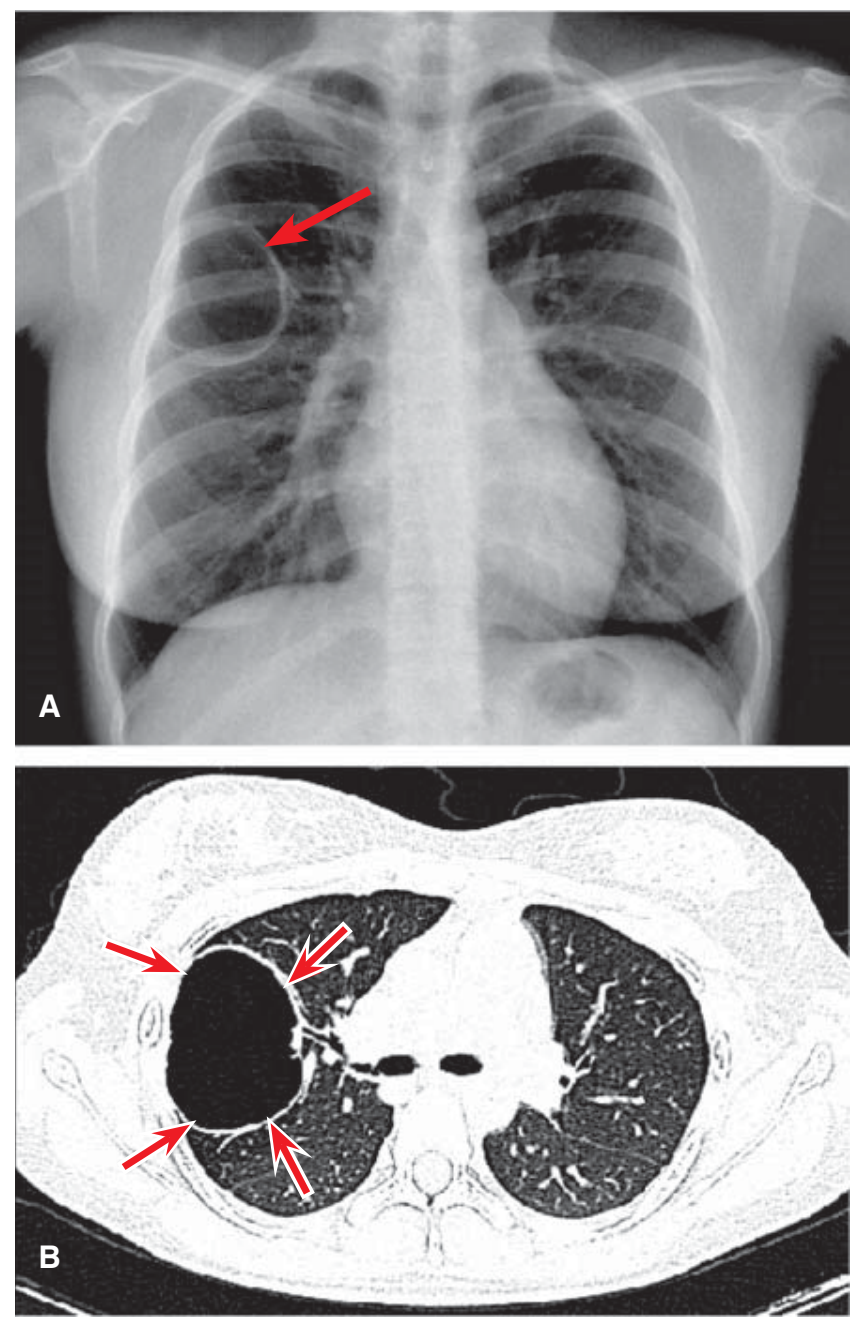

Figura 1. A. En la telerradiografía de tórax se observa lesión circular en hemitórax derecho (flecha). B. En este corte transversal de la TC de tórax se observa, delimitada por cuatro flechas rojas, la lesión en hemitórax derecho.

No hubo complicaciones. La inspección posoperatoria inmediata de la pieza quirúrgica reveló ausencia de contenido en su interior, revestimiento irregular de tipo fibrinoso, coloración verde amarillenta, no fétido (figuras 3A y 3B). La evolución posoperatoria fue satisfactoria retirándose el drenaje al primer día posoperatorio y el egreso hospitalario al segundo día.

\section{Anatomía patológica}

El estudio histopatológico describe elementos que constituyen la cápsula de un absceso organizado (antiguo): material fibrinohemático, cristales de colesterol, detritus celulares e infiltrado inflamatorio, además, áreas de destrucción pulmonar, fibrosis y neumonitis

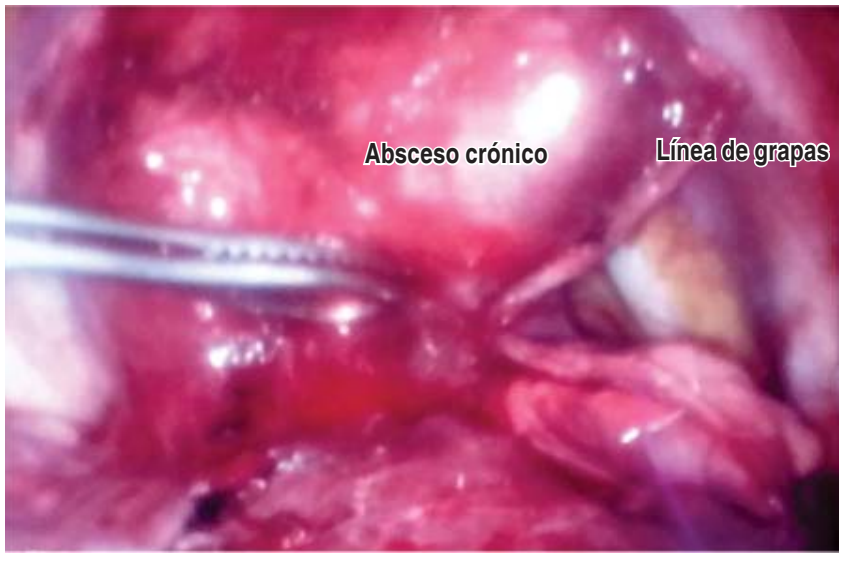

Figura 2. Imagen toracoscópica, al fondo la pared torácica; y en primer plano, el absceso crónico parcialmente resecado ya con una línea de grapas.

perilesionales, no observando piocitos que sustenten el diagnóstico de absceso activo. Concluye en absceso pulmonar crónico (figuras 4A y 4B).

Se realizó seguimiento clínico y radiológico hasta los primeros cinco meses posoperatorios con evolución satisfactoria (figuras $5 \mathrm{~A}$ y $5 \mathrm{~B}$ ). Cristales de colesterol y detritus celulares.

\section{DISCUSIÓN}

Dada la abundancia de términos existentes para designar las diferentes lesiones cavitadas del pulmón (quistes, bulas, ampollas, neumatoceles, cavidades, quistes aéreos, abscesos, bronquiectasias, etc.) y el empleo frecuente e incorrecto que de ellos se hace, algunos autores proponen denominar globalmente a este tipo de lesiones como espacios aéreos anómalos del pulmón o incluso como «agujeros» pulmonares. ${ }^{7}$

La cavitación es una zona definida de pérdida del parénquima, limitada por una pared y rellena de aire o líquido. Una cavidad es definida por la Sociedad Fleischner como el espacio lleno de aire dentro de una zona pulmonar de consolidación, de una masa o de un nódulo. Puede o no existir un nivel hidroaéreo. La mayor parte de las lesiones cavitadas son secundarias a la presencia de necrosis y a la expulsión del material necrótico por los bronquios. La demostración de un nivel hidroaéreo es el signo más consistente de la existencia de una lesión cavitada intrapulmonar. Así, el término cavitación implica necrosis y licuefacción del tejido pulmonar. Si el centro necrótico se comunica con el árbol traqueobronquial, el aire entraría a la cavidad y ello condiciona la apariencia translúcida dentro de la radiopacidad, algunas de las veces con nivel hidroaéreo. ${ }^{7,8}$ 

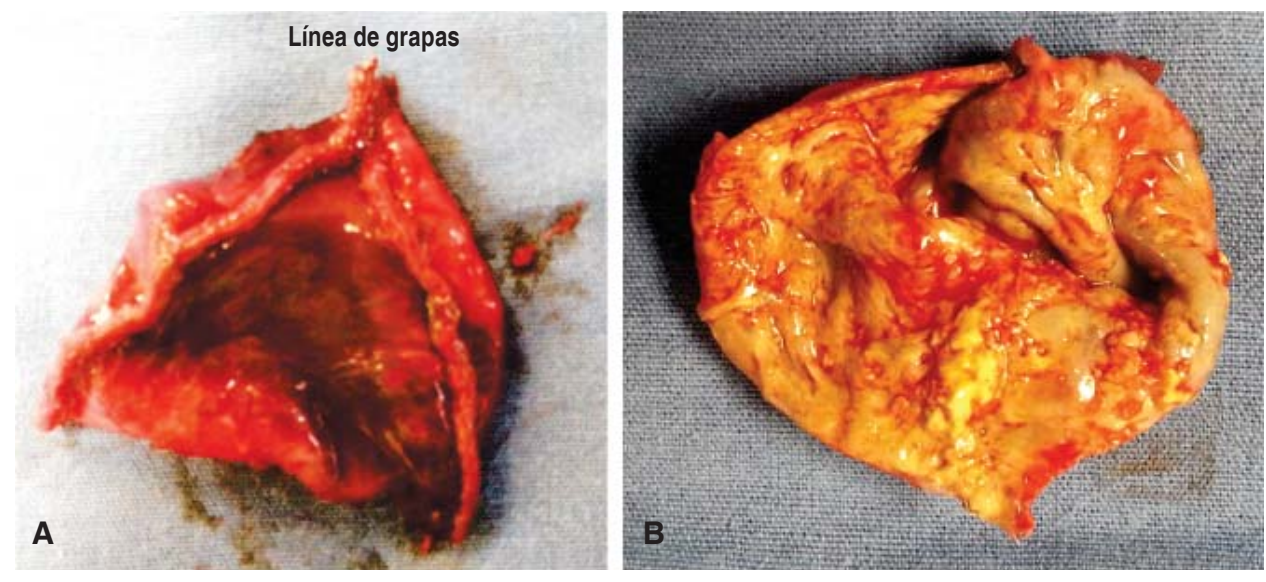

\section{Figura 3.}

A. Imagen macroscópica de la pieza quirúrgica. B. Imagen macroscópica al corte observando el interior del absceso crónico.
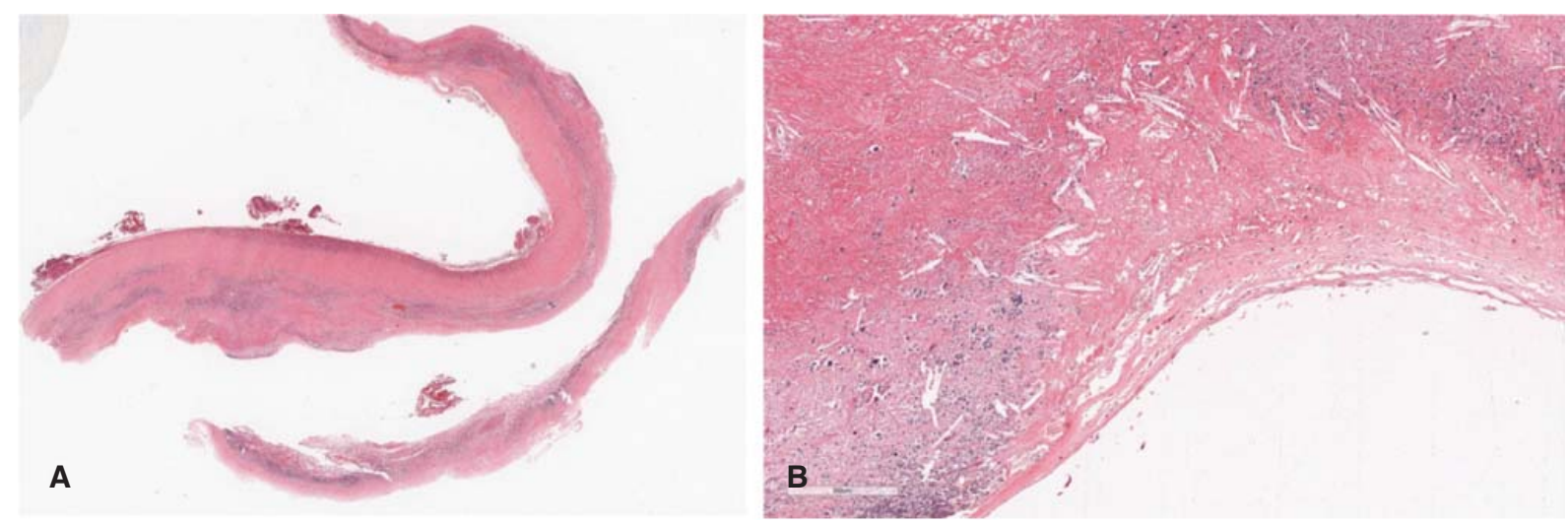

Figura 4. A. Fotomicrografía panorámica de los cortes histológicos de pared de la lesión constituida por tejido fibroso, material fibrinohemático e infiltrado inflamatorio. HyE 4x. B. Fotomicrografía que ilustra a mayor detalle la pared de la lesión. Se observa de arriba hacia abajo: tejido fibroso, fibrina, cristales de colesterol y detritus celulares, elementos que histológicamente constituyen la cápsula del absceso organizado (antiguo). No se observan piocitos que sustenten el diagnóstico de absceso activo. HyE 20x.
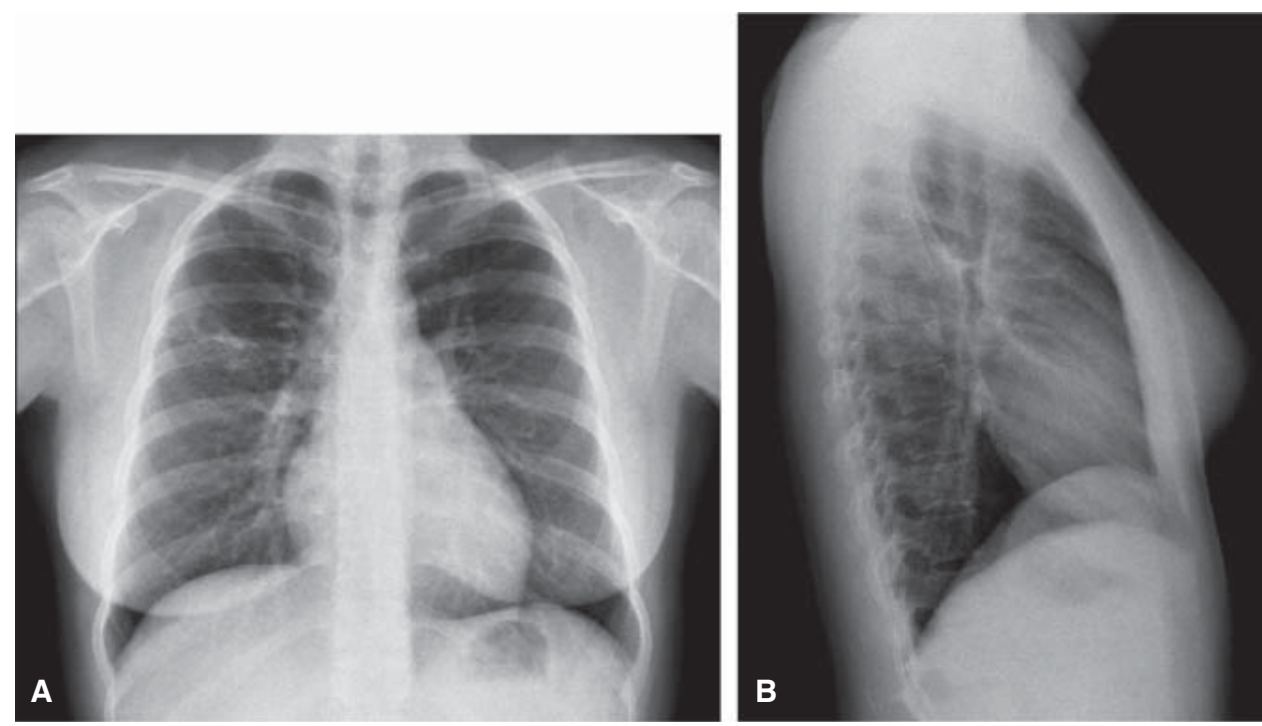

Figura 5.

A. Radiografía de tórax posteroanterior a los cinco meses del posquirúrgico, sin alteraciones. B. Radiografía de tórax lateral derecha a los cinco meses del posquirúrgico, sin alteraciones. 
Ante todo conviene aclarar que los conceptos de lesión cavitada y quiste no son sinónimos. Un quiste pulmonar no tiene por qué mostrar zonas de cavitación, mientras que una lesión destructiva pulmonar puede deberse a otras muchas causas al margen de la quística. Estrictamente, un quiste pulmonar se define como una lesión circunscrita, bien definida, mayor de $1 \mathrm{~cm}$ de diámetro, que contiene líquido o gas, de paredes finas y en cuya composición intervienen elementos celulares variables. Las lesiones quísticas, como se vio anteriormente, se cavitan cuando se expulsa, parcial o totalmente, su contenido al árbol bronquial. Todos los procesos quísticos del pulmón pueden sufrir cavitación. Sin embargo, los procesos auténticamente quísticos quedarían reducidos principalmente a la hidatidosis pulmonar, el quiste broncogénico, la malformación adenomatoidea quística congénita y otros. ${ }^{7}$

El análisis adecuado de las características semiológicas de una lesión cavitada (tamaño, grosor de la pared, revestimiento interno, contenido, localización, multiplicidad, alteraciones parenquimatosas circundantes), tanto en radiología simple, y sobre todo en TC de alta resolución, junto con la correcta valoración de los datos clínicos de que se disponga, puede limitar en gran manera las posibilidades diagnósticas de un determinado proceso destructivo del pulmón. ${ }^{9}$

Para nuestro caso, la valoración clínica aportó poco en la diferenciación entre una lesión quística o una cavitación pulmonar. No fue posible determinar claramente la sintomatología clásica de un absceso pulmonar (fiebre persistente de predominio nocturno y expectoración purulenta fétida). Al ingreso, la paciente estaba oligosintomática. Radiológicamente, está claro que el grosor de la pared no define el tipo de lesión; sin embargo, el hecho de que la pared de la lesión en la TC no superara los $4 \mathrm{~mm}$, la ausencia de nivel hidroaéreo y, sobre todo, la buena definición de sus bordes, nos hizo sospechar un origen quístico.

A favor de una lesión cavitada, un dato importante fue la comunicación de la lesión con el árbol bronquial, la cual se puede evidenciar claramente en la TC. Típicamente, un absceso pulmonar no se presenta con la imagen radiológica que presentamos. Más aún, la ausencia de consolidación perilesional nos hace dudar más de una cavidad de origen infeccioso; sin embargo, considerando la cronicidad de los síntomas, los múltiples esquemas antimicrobianos que recibió, y la clara comunicación con la vía aérea, consideramos que esta lesión, previamente ocupada por detritus celulares, material necrótico, y probablemente con un componente de consolidación perilesional, quedó desocupada tras la expectoración frecuente del material y como resultado del tratamiento recibido previamente. El reporte histo- patológico fue contundente al mencionar los elementos que constituyen la pared de la lesión (tejido fibroso, material fibrinohemático, cristales de colesterol, detritus celulares e infiltrado inflamatorio), las cuales la definen como una cavidad. Tras el reporte histopatológico, los autores concluyen en un absceso pulmonar crónico, y no un quiste ni una bula.

En la etiopatogenia de los espacios aéreos anómalos del pulmón se han involucrado, al margen del vaciamiento del contenido de las lesiones quísticas y de los procesos destructivos pulmonares de naturaleza congénita, a cinco mecanismos distintos: 1. Rotura de fibras elásticas del pulmón (ej.: enfisema pulmonar), 2. Dilatación bronquial (ej.: bronquiectasias), 3. Remodelación de la arquitectura pulmonar (ej.: fibrosis intersticial idiopática), 4. Multifactorial o desconocida (ej.: histiocitosis, síndrome de Klippel-Trenaunay), y finalmente, 5. Oclusión vascular o necrosis quística que sería la causa más común de destrucción pulmonar. Es típica de los infartos pulmonares sépticos y de las vasculitis infecciosas (micosis) y no infecciosas (Wegener). Esta última incluiría fenómenos de abscesificación por necrosis inflamatoria (tuberculosis, anaerobios, gramnegativos) ${ }^{7}$ correspondiente a este caso.

Los abscesos pulmonares fueron reconocidos y descritos desde la época de Hipócrates. ${ }^{10}$ Geppert los define como una infección pulmonar subaguda en la que la radiografía de tórax muestra una cavidad dentro del parénquima pulmonar. Se trata de una acumulación localizada de pus contenida dentro de la cavidad formada por la desintegración de los tejidos circundantes. ${ }^{11}$ La clasificación de los abscesos pulmonares está relacionada con el tiempo de evolución: abscesos agudos tienen menos de cuatro a seis semanas de evolución. Abscesos crónicos con más de seis semanas de inicio. Abscesos primarios, son originados de broncoaspiraciones o neumonías en un huésped normal. Abscesos secundarios son debidos a condiciones preexistentes de obstrucciones, propagación de la infección de sitios extrapulmonares, bronquiectasias o compromisos inmunes. ${ }^{4}$ Asumimos que el absceso se originó a partir de una neumonía de huésped normal no identificada o pobremente tratada, ya que no existe evidencia de otros factores de riesgo relacionados con broncoaspiración, obstrucción e inmunosupresión.

Los microorganismos anaerobios se encuentran presentes en el $89 \%$ de los pacientes. En estudios realizados por Bartlett et al., ${ }^{4} 46 \%$ de los cultivos de abscesos pulmonares tienen aislados únicamente anaerobios, mientras un $43 \%$ de cepas mixtas de aerobios y anaerobios. Los organismos aerobios relacionados con abscesos pulmonares o neumonías necrotizantes son S. aureus, Streptococcus pyogenes, Klebsiella pneumo- 
niae y $P$. aeruginosa. Menos frecuentes son los bacilos Gram (-) tales como E. coli y Haemophilus influenzae tipo $B$, los cuales pueden causar necrosis pulmonar. Neumonías cavitarias por organismos poco comunes son $N$. asteroides, Paragonimus westermani, Legionella species, Burkholderia pseudomallei y B. mallei, así como el Mycobacterium tuberculosis. Las infecciones fúngicas pueden causar cavitaciones en diabéticos y en huéspedes inmunocomprometidos (aspergilosis y mucoraceas). Entamoeba histolytica causa abscesos pulmonares casi siempre en la porción basal del lóbulo inferior derecho. No se aisló ningún germen en la pieza posoperatoria y esto puede estar relacionado a la utilización de múltiples esquemas antibióticos previos al ingreso a nuestra institución.

La presentación clínica de esta enfermedad puede ser coincidente con la presentación de las neumonías, o bien pueden ocurrir tardíamente en el curso clínico de la neumonía. La sospecha puede ser alta bajo condiciones predisponentes como broncoaspiración. Se deben considerar las siguientes situaciones: alcoholismo, alteraciones del estado de conciencia, efectos de anestésicos, disfagia o disfunción faríngea, gingivitis o piorrea, traumatismo cerrado o abierto de tórax, cirugía de tórax previa, obstrucción por neoplasias, bronquiectasias y embolismos pulmonares. El aliento fétido y las expectoraciones pútridas pueden ser notados; aunque la ausencia de éstas no excluye la posibilidad de una infección por anaerobios. Los pacientes con abscesos pulmonares primarios gradualmente desarrollan fiebre, tos, pleuritis o dolor en el hombro. ${ }^{12}$

Puede ser difícil distinguir con precisión entre los abscesos agudos y crónicos. Histológicamente, la cronicidad de la infección significa la formación de tejido de cicatrización maduro y el predominio de infiltración de células linfocíticas sobre polimorfonucleares. El absceso pulmonar crónico se caracteriza por aumento de la destrucción pulmonar y fibrosis del parénquima con extensa neumonitis, tal como reporta el estudio histopatológico de este caso. Algunos de estos cambios pueden ocurrir tan pronto como seis semanas después del inicio, si el tratamiento ha fracasado. ${ }^{13}$

Los signos de cronicidad pueden desarrollarse en un absceso que ha sido inadecuadamente drenado. Si bien no está demostrado estadísticamente, se cree que un verdadero infarto séptico que produce cavitación es menos probable que se resuelva después de drenaje quirúrgico, que un absceso que resulta de la aspiración. En el primer caso, puede ser que el parénquima circundante tenga un pobre suministro de sangre que es un factor determinante en el desarrollo de la cronicidad. Es cada vez más evidente que un absceso pulmonar crónico debe ser tratado en primer lugar por la resección pulmonar en lugar de por algún tipo de drenaje. ${ }^{13,14}$

Actualmente, sólo el $10 \%$ de los pacientes con absceso pulmonar requieren cirugía. Algunas indicaciones específicas incluyen: tratamiento médico fallido en un período de ocho semanas, sospecha de cáncer, complicaciones propias del absceso como empiema o fístula broncopleural, hemoptisis masiva, persistencia de una cavidad mayor de $6 \mathrm{~cm}$ de diámetro después de ocho semanas de tratamiento médico. ${ }^{10}$ La decisión quirúrgica en este caso fue la ausencia de respuesta a tratamiento médico y el tamaño de la lesión.

\section{Ventajas y desventajas del abordaje toracoscópico}

En relación con el abordaje toracoscópico y comparado con la vía clásica, las ventajas más importantes son: la disminución del dolor, el acortamiento de la hospitalización y un mejor resultado estético. Otras ventajas adicionales son la participación de todo el equipo en las decisiones, puesto que la observación del procedimiento en un monitor facilita el intercambio de opiniones. También es útil como elemento de docencia, al permitir la grabación de las intervenciones. Asimismo, son ventajas indiscutibles la amplificación de la imagen y la posibilidad de explorar toda la cavidad torácica, incluso aquellos lugares tradicionalmente considerados como «ciegos» en la cirugía abierta.

Entre las desventajas se puede destacar que al ser relativamente nuevo en nuestro medio, requiere una curva de aprendizaje, por lo que en un principio la duración de la operación es mayor y pueden ocurrir contratiempos, problemas que deben disminuir en la medida que el equipo operador adquiera mayor experiencia. El costo, dado el uso de instrumental nuevo y sofisticado, también es superior, pero tiende a disminuir con el uso rutinario. ${ }^{15}$

\section{REFERENCIAS}

1. Tuddenham WJ. Glossary of terms for thoracic radiology: recommendations of the Nomenclature Committee of the Fleischner Society. AJR Am J Roentgenol 1984;143(3):509-517.

2. Naidich DP, Webb WR, Müller NL, Vlahos I, Krinsky GA, Kim EE. Computed tomography and magnetic resonance of the thorax. J Nucl Med 2007;48(12):2088.

3. Ryu JH, Swensen SJ. Cystic and cavitary lung diseases: focal and diffuse. Mayo Clin Proc 2003;78(6):744-752.

4. Bartlett JG. Lung abscess and necrotizing pneumonia. In: Gorbach SL, Bartlett JG, Blacklow NR, editors. Infectious diseases. 3rd ed. Philadelphia: Saunders; 1992.p.105-211. 
5. Bloomberg AE. Thoracoscopy in perspective. Surg Gynecol Obstet 1978;147(3):433-443.

6. Boutin C, Loddenkemper R, Astoul P. Diagnostic and therapeutic thoracoscopy: techniques and indications in pulmonary medicine. Tuber Lung Dis 1993;74(4):225239.

7. Pedrosa C, Casanova R. Diagnóstico por imagen. Vol. I: Tórax. 3ra ed. Madrid: Marban; 2009.

8. Armstrong P, Wilson AG, Dee P, Hansell DM. Imaging of diseases of the chest. 2nd ed. St. Louis: Mosby; 1995.

9. Motta-Ramírez GA, García-Arayza MG, Ortiz-León JL, Castillo-Lima JA. Detección radiológica y por imagen de lesiones pulmonares cavitadas. Abordaje para el médico general. Rev Sanid Milit Mex 2008;62(4):174-186.

10. Wiedemann HP, Rice TW. Lung abscess and empyema. Semin Thorac Cardiovasc Surg 1995;7(2):119-128.

11. Niederman MS, Sacosi GA, Glassroth J, editors. Respiratory infections. Philadelphia: Saunders; 1994.

12. Finegold MS, Fishman AV. Empyema and lung abscess. In: Fishman's pulmonary disease and disorders. New York: MacGraw-Hill; 1998.p.2021-2033.
13. Samson PC, Dugan DJ. The management of acute and chronic pulmonary abscess. Calif Med 1948;69(5):364366.

14. Lindskog GE, Alley RD. Bilateral bronchiectasis; an analysis of 43 consecutive cases. Arch Surg 1950;60(3):465472.

15. Hazelrigg SR, Nunchuck SK, LoCicero J 3rd. Video Assisted Thoracic Surgery Study Group data. Ann Thorac Surg 1993;56(5):1039-1043.

\section{$\triangle$ Correspondencia:}

Dr. Víctor $\mathrm{H}$. Zotes Valdivia

Servicio de Cirugía Torácica, Instituto Nacional de Enfermedades Respiratorias Ismael Cosío Villegas. Calzada de Tlalpan Núm. 4502,

Colonia Sección XVI, 14080, México, D.F. Teléfono: (55) 54871700, extensión 5210

Correo electrónico: drzotesvaldivia@hotmail.com; markcardio@hotmail.com

Los autores declaran no tener conflicto de intereses. 